\title{
On the Recurrence Properties of Generalized Tribonacci Sequence
}

\author{
Yüksel Soykan \\ Department of Mathematics, Art and Science Faculty, \\ Zonguldak Bülent Ecevit University, 67100, Zonguldak, Turkey \\ e-mail: yuksel_soykan@hotmail.com
}

\begin{abstract}
In this paper, we investigate the recurrence properties of the generalized Tribonacci sequence and present how the generalized Tribonacci sequence at negative indices can be expressed by the sequence itself at positive indices.
\end{abstract}

\section{Introduction}

In 2021, Lin 8 stated on page 4 of 12 that "Recently, Professor Tianxin Cai visited Northwest University and gave a talk about a series of linear recurrence sequences and their properties, which incited our interest in this field. There are many recursive identities concerning the Fibonacci, Tribonacci, and Lucas sequences, etc. However, few studies have been conducted regarding the Narayana sequence. Professor Cai proposed an open problem: Whether and how can the Narayana sequence at negative indices be expressed by the sequence itself at positive indices?" Then Lin presented the following theorem as a main result and proved it:

Theorem 1. For $n \in \mathbb{Z}$, we have

$$
N_{-n}=2 N_{n}^{2}+N_{2 n}-3 N_{n+1} N_{n}
$$

Received: February 23, 2021; Accepted: March 12, 2021

2020 Mathematics Subject Classification: 11B37, 11B39, $11 B 83$.

Keywords and phrases: Tribonacci numbers, Narayana numbers, Padovan numbers, negative indices, recurrence relations. 
Here, Narayana's cows sequence $\left\{N_{n}\right\}$ satisfies a third-order recurrence relation:

$$
N_{n}=N_{n-1}+N_{n-3}, \text { for } n \geq 3
$$

with the initial values $N_{0}=0, N_{2}=1, N_{2}=1$. It can be extended to negative indices by defining

$$
N_{-n}=-N_{-(n-2)}+N_{-(n-3)}, \text { for } n=1,2,3, \ldots
$$

Lin also stated on the same page that "Theorem 1 solves Professor Cai's problem completely. It illustrates the connection between the Narayana sequence at the positive index and the negative index. By Theorem 1, we obtain the recurrence property of the sequence at the negative index, which deepens our knowledge of the nature of the sequence."

Now, we can propose an open problem as follows: Whether and how can the generalized Tribonacci sequence $W_{n}$ at negative indices be expressed by the sequence itself at positive indices?

We present our main result as follows which completely solves the above problem for the generalized Tribonacci sequence $W_{n}$.

Theorem 2. For $n \in \mathbb{Z}$, we have

$$
W_{-n}=t^{-n}\left(W_{2 n}-H_{n} W_{n}+\frac{1}{2}\left(H_{n}^{2}-H_{2 n}\right) W_{0}\right) .
$$

Note that $H_{n}$ can be written in terms of $W_{n}$ using Lemma 4 below. Next, we recall the definitions of generalized Tribonacci sequence $W_{n}$ and its two special cases, namely $(r, s, t)$ sequence $G_{n}$ and $(r, s, t)$ Lucas sequence $H_{n}$. The generalized $(r, s, t)$ sequence (or generalized Tribonacci sequence or generalized 3 -step Fibonacci sequence) $\left\{W_{n}\left(W_{0}, W_{1}, W_{2} ; r, s, t\right)\right\}_{n \geq 0}$ (or shortly $\left\{W_{n}\right\}_{n \geq 0}$ ) is defined as follows:

$$
W_{n}=r W_{n-1}+s W_{n-2}+t W_{n-3}, \quad W_{0}=a, W_{1}=b, W_{2}=c, \quad n \geq 3
$$

where $W_{0}, W_{1}, W_{2}$ are arbitrary complex (or real) numbers and $r, s, t$ are real numbers. This sequence has been studied by many authors, see for example 1,2 , 3, 4, 5, 7, 9, 10, 11, 13, 22, 24, 25. 
The sequence $\left\{W_{n}\right\}_{n \geq 0}$ can be extended to negative subscripts by defining

$$
W_{-n}=-\frac{s}{t} W_{-(n-1)}-\frac{r}{t} W_{-(n-2)}+\frac{1}{t} W_{-(n-3)}
$$

for $n=1,2,3, \ldots$ when $t \neq 0$. Therefore, recurrence (1.1) holds for all integer $n$.

In the following Table 1 we present some special cases of generalized Tribonacci sequence.

Table 1. A few special case of generalized Tribonacci sequences.

\begin{tabular}{ccc}
\hline No & Sequences (Numbers) & Notation \\
\hline 1 & Generalized Tribonacci & $\left\{V_{n}\right\}=\left\{W_{n}\left(W_{0}, W_{1}, W_{2} ; 1,1,1\right)\right\}$ \\
2 & Generalized Third Order Pell & $\left\{V_{n}\right\}=\left\{W_{n}\left(W_{0}, W_{1}, W_{2} ; 2,1,1\right)\right\}$ \\
3 & Generalized Padovan & $\left\{V_{n}\right\}=\left\{W_{n}\left(W_{0}, W_{1}, W_{2} ; 0,1,1\right)\right\}$ \\
4 & Generalized Pell-Padovan & $\left\{V_{n}\right\}=\left\{W_{n}\left(W_{0}, W_{1}, W_{2} ; 0,2,1\right)\right\}$ \\
5 & Generalized Jacobsthal-Padovan & $\left\{V_{n}\right\}=\left\{W_{n}\left(W_{0}, W_{1}, W_{2} ; 0,1,2\right)\right\}$ \\
6 & Generalized Narayana & $\left\{V_{n}\right\}=\left\{W_{n}\left(W_{0}, W_{1}, W_{2} ; 1,0,1\right)\right\}$ \\
7 & Generalized Third Order Jacobsthal & $\left\{V_{n}\right\}=\left\{W_{n}\left(W_{0}, W_{1}, W_{2} ; 1,1,2\right)\right\}$ \\
8 & Generalized 3-primes & $\left\{V_{n}\right\}=\left\{W_{n}\left(W_{0}, W_{1}, W_{2} ; 2,3,5\right)\right\}$ \\
9 & Generalized Reverse 3-primes & $\left\{V_{n}\right\}=\left\{W_{n}\left(W_{0}, W_{1}, W_{2} ; 5,3,2\right)\right\}$ \\
\hline
\end{tabular}


In literature, for example, the following names and notations (see Table 2) are used for the special case of $r, s, t$ and initial values.

Table 2. A few special case of generalized $(r, s, t)$ (generalized Tribonacci) sequence

\begin{tabular}{|c|c|c|c|c|}
\hline No & Sequences (Numbers) & Notation & OEIS 12$]$ & Reference \\
\hline 1 & Tribonacci & $\left\{T_{n}\right\}=\left\{W_{n}(0,1,1 ; 1,1,1)\right\}$ & $\mathrm{A} 000073, \mathrm{~A} 057597$ & 14 \\
\hline 2 & Tribonacci-Lucas & $\left\{K_{n}\right\}=\left\{W_{n}(3,1,3 ; 1,1,1)\right\}$ & $\mathrm{A} 001644, \mathrm{~A} 073145$ & 14 \\
\hline 3 & Tribonacci-Perrin & $\left\{M_{n}\right\}=\left\{W_{n}(3,0,2 ; 1,1,1)\right\}$ & & 14 \\
\hline 4 & modified Tribonacci & $\left\{U_{n}\right\}=\left\{W_{n}(1,1,1 ; 1,1,1)\right\}$ & & 14 \\
\hline 5 & modified Tribonacci-Lucas & $\left\{G_{n}\right\}=\left\{W_{n}(4,4,10 ; 1,1,1)\right\}$ & & 14 \\
\hline 6 & adjusted Tribonacci-Lucas & $\left\{H_{n}\right\}=\left\{W_{n}(4,2,0 ; 1,1,1)\right\}$ & & 14 \\
\hline 7 & third order Pell & $\left\{P_{n}^{(3)}\right\}=\left\{W_{n}(0,1,2 ; 2,1,1)\right\}$ & A077939, A077978 & 15 \\
\hline 8 & third order Pell-Lucas & $\left\{Q_{n}^{(3)}\right\}=\left\{W_{n}(3,2,6 ; 2,1,1)\right\}$ & A 276225, A 276228 & 15 \\
\hline 9 & third order modified Pell & $\left\{E_{n}^{(3)}\right\}=\left\{W_{n}(0,1,1 ; 2,1,1)\right\}$ & A077997, A078049 & 15 \\
\hline 10 & third order Pell-Perrin & $\left\{R_{n}^{(3)}\right\}=\left\{W_{n}(3,0,2 ; 2,1,1)\right\}$ & & 22 \\
\hline 11 & Padovan (Cordonnier) & $\left\{P_{n}\right\}=\left\{W_{n}(1,1,1 ; 0,1,1)\right\}$ & A000931 & 16 \\
\hline 12 & Perrin (Padovan-Lucas) & $\left\{E_{n}\right\}=\left\{W_{n}(3,0,2 ; 0,1,1)\right\}$ & A 001608, A 078712 & $\overline{16}$ \\
\hline 13 & Padovan-Perrin & $\left\{S_{n}\right\}=\left\{W_{n}(0,0,1 ; 0,1,1)\right\}$ & A000931, A176971 & \begin{tabular}{|l|l|}
16 \\
\end{tabular} \\
\hline 14 & modified Padovan & $\left\{A_{n}\right\}=\left\{W_{n}(3,1,3 ; 0,1,1)\right\}$ & & 16 \\
\hline 15 & adjusted Padovan & $\left\{U_{n}\right\}=\left\{W_{n}(0,1,0 ; 0,1,1)\right\}$ & & 22 \\
\hline 16 & Pell-Padovan & $\left\{R_{n}\right\}=\left\{W_{n}(1,1,1 ; 0,2,1)\right\}$ & A066983, A128587 & 17 \\
\hline 17 & Pell-Perrin & $\left\{C_{n}\right\}=\left\{W_{n}(3,0,2 ; 0,2,1)\right\}$ & & 17 \\
\hline 18 & third order Fibonacci-Pell & $\left\{G_{n}\right\}=\left\{W_{n}(1,0,2 ; 0,2,1)\right\}$ & & 17 \\
\hline 19 & third order Lucas-Pell & $\left\{B_{n}\right\}=\left\{W_{n}(3,0,4 ; 0,2,1)\right\}$ & & 17 \\
\hline 20 & adjusted Pell-Padovan & $\left\{M_{n}\right\}=\left\{W_{n}(0,1,0 ; 0,2,1)\right\}$ & & 22 \\
\hline 21 & Jacobsthal-Padovan & $\left\{Q_{n}\right\}=\left\{W_{n}(1,1,1 ; 0,1,2)\right\}$ & A159284 & 18 \\
\hline 22 & Jacobsthal-Perrin (-Lucas) & $\left\{L_{n}\right\}=\left\{W_{n}(3,0,2 ; 0,1,2)\right\}$ & A072328 & 18 \\
\hline 23 & adjusted Jacobsthal-Padovan & $\left\{K_{n}\right\}=\left\{W_{n}(0,1,0 ; 0,1,2)\right\}$ & & 18 \\
\hline 24 & modified Jacobsthal-Padovan & $\left\{M_{n}\right\}=\left\{W_{n}(3,1,3 ; 0,1,2)\right\}$ & & $\overline{18}$ \\
\hline 25 & Narayana & $\left\{N_{n}\right\}=\left\{W_{n}(0,1,1 ; 1,0,1)\right\}$ & A078012 & 19 \\
\hline 26 & Narayana-Lucas & $\left\{U_{n}\right\}=\left\{W_{n}(3,1,1 ; 1,0,1)\right\}$ & A001609 & 19 \\
\hline 27 & Narayana-Perrin & $\left\{H_{n}\right\}=\left\{W_{n}(3,0,2 ; 1,0,1)\right\}$ & & 19 \\
\hline 28 & third order Jacobsthal & $\left\{J_{n}^{(3)}\right\}=\left\{W_{n}(0,1,1 ; 1,1,2)\right\}$ & A077947 & 20 \\
\hline 29 & third order Jacobsthal-Lucas & $\left\{j_{n}^{(3)}\right\}=\left\{W_{n}(2,1,5 ; 1,1,2)\right\}$ & A226308 & 20 \\
\hline 30 & modified third order Jacobsthal-Lucas & $\left\{K_{n}^{(3)}\right\}=\left\{W_{n}(3,1,3 ; 1,1,2)\right\}$ & & 20 \\
\hline 31 & third order Jacobsthal-Perrin & $\left\{Q_{n}^{(3)}\right\}=\left\{W_{n}(3,0,2 ; 1,1,2)\right\}$ & & 20 \\
\hline 32 & 3-primes & $\left\{G_{n}\right\}=\left\{W_{n}(0,1,2 ; 2,3,5)\right\}$ & & 21 \\
\hline 33 & Lucas 3-primes & $\left\{H_{n}\right\}=\left\{W_{n}(3,2,10 ; 2,3,5)\right\}$ & & 21 \\
\hline 34 & modified 3-primes & $\left\{E_{n}\right\}=\left\{W_{n}(0,1,1 ; 2,3,5)\right\}$ & & 21 \\
\hline 35 & reverse 3 -primes & $\left\{N_{n}\right\}=\left\{W_{n}(0,1,5 ; 5,3,2)\right\}$ & & 23 \\
\hline 36 & reverse Lucas 3-primes & $\left\{S_{n}\right\}=\left\{W_{n}(3,5,31 ; 5,3,2)\right\}$ & & 23 \\
\hline 37 & reverse modified 3 -primes & $\left\{U_{n}\right\}=\left\{W_{n}(0,1,4 ; 5,3,2)\right\}$ & & 23 \\
\hline
\end{tabular}

Here, OEIS stands for On-line Encyclopedia of Integer Sequences. For easy writing, from now on, we drop the superscripts from the sequences, for example we write $J_{n}$ for $J_{n}^{(3)}$. 
It is well known that the generalized $(r, s, t)$ numbers (the generalized Tribonacci numbers) can be expressed, for all integers $n$, using Binet's formula

$$
W_{n}=A_{1} \alpha^{n}+A_{2} \beta^{n}+A_{3} \gamma^{n}
$$

where

$$
\begin{aligned}
A_{1}=\frac{W_{2}-(\beta+\gamma) W_{1}+\beta \gamma W_{0}}{(\alpha-\beta)(\alpha-\gamma)}, & A_{2}=\frac{W_{2}-(\alpha+\gamma) W_{1}+\alpha \gamma W_{0}}{(\beta-\alpha)(\beta-\gamma)}, \\
A_{3} & =\frac{W_{2}-(\alpha+\beta) W_{1}+\alpha \beta W_{0}}{(\gamma-\alpha)(\gamma-\beta)} .
\end{aligned}
$$

and $\alpha, \beta, \gamma$ are the roots of characteristic equation of $W_{n}$ which is given by

$$
x^{3}-r x^{2}-s x-t=0
$$

Note that we have the following identities

$$
\left\{\begin{array}{c}
\alpha+\beta+\gamma=r, \\
\alpha \beta+\alpha \gamma+\beta \gamma=-s, \\
\alpha \beta \gamma=t .
\end{array}\right.
$$

Note that the Binet form of a sequence satisfying (1.3) for non-negative integers is valid for all integers $n$. Now we define two special cases of the generalized $(r, s, t)$ sequence $\left\{W_{n}\right\}$. (r, s,t) sequence $\left\{G_{n}\right\}_{n \geq 0}$ and Lucas $(r, s, t)$ sequence $\left\{H_{n}\right\}_{n \geq 0}$ are defined, respectively, by the third-order recurrence relations

$$
\begin{array}{ll}
G_{n+3}=r G_{n+2}+s G_{n+1}+t G_{n}, & G_{0}=0, G_{1}=1, G_{2}=r, \\
H_{n+3}=r H_{n+2}+s H_{n+1}+t H_{n}, & H_{0}=3, H_{1}=r, H_{2}=2 s+r^{2},
\end{array}
$$

The sequences $\left\{G_{n}\right\}_{n \geq 0}$ and $\left\{H_{n}\right\}_{n \geq 0}$ can be extended to negative subscripts by defining

$$
\begin{aligned}
G_{-n} & =-\frac{s}{t} G_{-(n-1)}-\frac{r}{t} G_{-(n-2)}+\frac{1}{t} G_{-(n-3)}, \\
H_{-n} & =-\frac{s}{t} H_{-(n-1)}-\frac{r}{t} H_{-(n-2)}+\frac{1}{t} H_{-(n-3)},
\end{aligned}
$$

for $n=1,2,3, \ldots$ respectively. Some special cases of $(r, s, t)$ sequence $\left\{G_{n}(0,1, r ; r, s, t)\right\}_{n \geq 0}$ and Lucas $(r, s, t)$ sequence $\left\{H_{n}\left(3, r, 2 s+r^{2} ; r, s, t\right)\right\}_{n \geq 0}$ are as follows: 
1. $G_{n}(0,1,1 ; 1,1,1)=T_{n}$, Tribonacci sequence,

2. $H_{n}(3,1,3 ; 1,1,1)=K_{n}$, Tribonacci-Lucas sequence,

3. $G_{n}(0,1,2 ; 2,1,1)=P_{n}$, third order Pell sequence,

4. $H_{n}(3,2,6 ; 2,1,1)=Q_{n}$, third order Pell-Lucas sequence,

5. $G_{n}(0,1,0 ; 0,1,1)=U_{n}$, adjusted Padovan sequence,

6. $H_{n}(3,0,2 ; 0,1,1)=E_{n}$, Perrin (Padovan-Lucas) sequence,

7. $G_{n}(0,1,0 ; 0,2,1)=M_{n}$, adjusted Pell-Padovan sequence

8. $H_{n}(3,0,4 ; 0,2,1)=B_{n}$, third order Lucas-Pell sequence,

9. $G_{n}(0,1,0 ; 0,1,2)=K_{n}$, adjusted Jacobsthal-Padovan sequence,

10. $H_{n}(3,0,2 ; 0,1,2)=L_{n}$, Jacobsthal-Perrin (-Lucas) sequence,

11. $G_{n}(0,1,1 ; 1,0,1)=N_{n}$, Narayana sequence,

12. $H_{n}(3,1,1 ; 1,0,1)=U_{n}$, Narayana-Lucas sequence,

13. $G_{n}(0,1,1 ; 1,1,2)=J_{n}$, third order Jacobsthal sequence,

14. $H_{n}(3,1,3 ; 1,1,2)=K_{n}$, modified third order Jacobsthal-Lucas sequence,

15. $G_{n}(0,1,2 ; 2,3,5)=G_{n}, 3$-primes sequence,

16. $H_{n}(3,2,10 ; 2,3,5)=H_{n}$, Lucas 3 -primes sequence.

17. $G_{n}(0,1,5 ; 5,3,2)=N_{n}$, reverse 3 -primes sequence,

18. $H_{n}(3,5,31 ; 5,3,2)=S_{n}$, reverse Lucas 3-primes sequence.

For all integers $n,(r, s, t)$ and Lucas $(r, s, t)$ numbers can be expressed using Binet's formulas as

$$
\begin{aligned}
G_{n} & =\frac{\alpha^{n+1}}{(\alpha-\beta)(\alpha-\gamma)}+\frac{\beta^{n+1}}{(\beta-\alpha)(\beta-\gamma)}+\frac{\gamma^{n+1}}{(\gamma-\alpha)(\gamma-\beta)}, \\
H_{n} & =\alpha^{n}+\beta^{n}+\gamma^{n},
\end{aligned}
$$

respectively. 


\section{The Proof of Theorem 2}

To prove Theorem 2, we need following lemma.

Lemma 3. For $n \in \mathbb{Z}$, denote

$$
S_{n}=\alpha^{n} \beta^{n}+\alpha^{n} \gamma^{n}+\beta^{n} \gamma^{n}
$$

where $\alpha, \beta$ and $\gamma$ are as in defined in Formula (1.4). Then the followings hold:

(a) For $n \in \mathbb{Z}$, we have $S_{n}=t^{n} H_{-n}$ and $S_{-n}=t^{-n} H_{n}$.

(b) $S_{n}$ has the recurrence relation so that

$$
S_{n}=-s S_{n-1}-r t S_{n-2}+t^{2} S_{n-3}
$$

with the initial conditions $S_{0}=3, S_{1}=-s, S_{2}=s^{2}-2 r t$. The sequence at negative indices is given by

$$
S_{-n}=-\frac{-r t}{t^{2}} S_{-(n-1)}-\frac{-s}{t^{2}} S_{-(n-2)}+\frac{1}{t^{2}} S_{-(n-3)}, \text { for } n=1,2,3, \ldots .
$$

(c) $S_{n}$ has the identity so that

$$
S_{n}=\frac{1}{2}\left(H_{n}^{2}-H_{2 n}\right)
$$

Proof.

(a) From the definition of $S_{n}$ and $H_{n}$, we obtain

$$
t^{n} H_{-n}=\alpha^{-n} t^{n}+\beta^{-n} t^{n}+\gamma^{-n} t^{n}=\alpha^{n} \beta^{n}+\alpha^{n} \gamma^{n}+\beta^{n} \gamma^{n}=S_{n}
$$

i.e., $S_{n}=t^{n} H_{-n}$ and so $S_{-n}=t^{-n} H_{n}$.

(b) With Formula (1.4) or using the formula $S_{n}=(-u)^{n} H_{-n}$, we obtain initial values of $S_{n}$ as

$$
\begin{aligned}
S_{0} & =t^{0} H_{0}=3, \\
S_{1} & =t^{1} H_{-1}=t \times\left(-\frac{s}{t}\right)=-s \\
S_{2} & =t^{2} H_{-2}=t^{2} \times \frac{1}{t^{2}}\left(s^{2}-2 r t\right)=s^{2}-2 r t,
\end{aligned}
$$


or

$S_{2}=\alpha^{2} \beta^{2}+\alpha^{2} \gamma^{2}+\beta^{2} \gamma^{2}=(\alpha \beta+\alpha \gamma+\beta \gamma)^{2}-2 \alpha \beta \gamma(\alpha+\beta+\gamma)=s^{2}-2 r t$

For $n \geq 3$, we have

$$
\begin{aligned}
S_{1} S_{n-1}= & \left(\alpha^{n} \beta^{n}+\alpha^{n} \gamma^{n}+\beta^{n} \gamma^{n}\right)+\alpha \beta \gamma\left(\alpha^{n-2} \beta^{n-2}(\alpha+\beta)\right. \\
& \left.+\alpha^{n-2} \gamma^{n-2}(\alpha+\gamma)+\beta^{n-2} \gamma^{n-2}(\beta+\gamma)\right) \\
= & S_{n}+r t S_{n-2}-t^{2} S_{n-3}=(-s) S_{n-1} .
\end{aligned}
$$

(c) From the definition of $S_{n}$ we get

$$
2 S_{n}=\left(\alpha^{n}+\beta^{n}+\gamma^{n}\right)^{2}-\left(\alpha^{2 n}+\beta^{2 n}+\gamma^{2 n}\right)=H_{n}^{2}-H_{2 n} .
$$

Now, we shall complete the proof of Theorem 2 .

\section{The Proof of Theorem 2:}

Using our Lemma 3 (i.e., $S_{n}=t^{n} H_{-n}=\frac{1}{2}\left(H_{n}^{2}-H_{2 n}\right)$ ) and taking $m=-n$ in Howard [6. Identity (1.5)] we obtain Theorem 2, For completeness, we give the detailed proof. For $n \in \mathbb{Z}$, we have

$$
\begin{aligned}
W_{n} H_{n}= & \left(A_{1} \alpha^{n}+A_{2} \beta^{n}+A_{3} \gamma^{n}\right)\left(\alpha^{n}+\beta^{n}+\gamma^{n}\right) \\
= & W_{2 n}+\left(A_{1}+A_{2}+A_{3}\right) \alpha^{n} \beta^{n}+\left(A_{1}+A_{3}+A_{2}\right) \alpha^{n} \gamma^{n} \\
& +\left(A_{2}+A_{3}+A_{1}\right) \beta^{n} \gamma^{n}-\left(A_{3} \alpha^{n} \beta^{n}+A_{2} \alpha^{n} \gamma^{n}+A_{1} \beta^{n} \gamma^{n}\right) \\
= & W_{2 n}+\left(A_{1}+A_{2}+A_{3}\right)\left(\alpha^{n} \beta^{n}+\alpha^{n} \gamma^{n}+\beta^{n} \gamma^{n}\right) \\
& -\left(A_{3} \alpha^{n} \beta^{n}+A_{2} \alpha^{n} \gamma^{n}+A_{1} \beta^{n} \gamma^{n}\right) \\
= & W_{2 n}+W_{0} S_{n}-t^{n} W_{-n} .
\end{aligned}
$$

By Lemma 3 (c), it follows that

$$
W_{n} H_{n}=W_{2 n}+\frac{1}{2}\left(H_{n}^{2}-H_{2 n}\right) W_{0}-t^{n} W_{-n}
$$

and so

$$
W_{-n}=t^{-n}\left(W_{2 n}-H_{n} W_{n}+\frac{1}{2}\left(H_{n}^{2}-H_{2 n}\right) W_{0}\right)
$$

Now, we present a basic relation between $\left\{H_{n}\right\}$ and $\left\{W_{n}\right\}$ which can be used to write $H_{n}$ in terms of $W_{n}$. 
Lemma 4. The following equality is true:

$\left(W_{2}^{3}+(t+r s) W_{1}^{3}+t^{2} W_{0}^{3}+\left(r^{2}-s\right) W_{1}^{2} W_{2}-2 r W_{1} W_{2}^{2}-s W_{0} W_{2}^{2}+r t W_{0}^{2} W_{2}+\right.$ $\left.\left(s^{2}+r t\right) W_{0} W_{1}^{2}+2 s t W_{0}^{2} W_{1}+(r s-3 t) W_{0} W_{1} W_{2}\right) H_{n}=\left(3 W_{2}^{2}+\left(r^{2}-s\right) W_{1}^{2}+r t W_{0}^{2}-\right.$ $\left.4 r W_{1} W_{2}-2 s W_{0} W_{2}+(r s-3 t) W_{0} W_{1}\right) W_{n+2}+\left(-2 r W_{2}^{2}+3 t W_{1}^{2}-2 s W_{1} W_{2}-3 t W_{0} W_{2}+\right.$ $\left.3 r s W_{1}^{2}+2 s t W_{0}^{2}+2 r^{2} W_{1} W_{2}+2 s^{2} W_{0} W_{1}+r s W_{0} W_{2}+2 r t W_{0} W_{1}\right) W_{n+1}+\left(-s W_{2}^{2}+\right.$ $\left.\left(s^{2}+r t\right) W_{1}^{2}+3 t^{2} W_{0}^{2}+(r s-3 t) W_{1} W_{2}+2 r t W_{0} W_{2}+4 s t W_{0} W_{1}\right) W_{n}$.

Proof. It is given in Soykan 22.

Next, we present a remark which presents how $H_{n}$ can be written in terms of $W_{n}$.

Remark 5. To express $W_{-n}$ by the sequence itself at positive indices we need that $H_{n}$ can be written in terms of $W_{n}$. For this, writing

$$
H_{n}=a \times W_{n+2}+b \times W_{n+1}+c \times W_{n}
$$

and solving the system of equations

$$
\begin{aligned}
& H_{0}=a \times W_{2}+b \times W_{1}+c \times W_{0} \\
& H_{1}=a \times W_{3}+b \times W_{2}+c \times W_{1} \\
& H_{2}=a \times W_{4}+b \times W_{3}+c \times W_{2}
\end{aligned}
$$

or

$$
\left(\begin{array}{l}
a \\
b \\
c
\end{array}\right)=\left(\begin{array}{lll}
W_{2} & W_{1} & W_{0} \\
W_{3} & W_{2} & W_{1} \\
W_{4} & W_{3} & W_{2}
\end{array}\right)^{-1}\left(\begin{array}{c}
H_{0} \\
H_{1} \\
H_{2}
\end{array}\right)
$$

we find $a, b, c$ so that $H_{n}$ can be written in terms of $W_{n}$ and we can replace this $H_{n}$ in Theorem 2 ,

Using Theorem 2 and Lemma 4 or Remark 5 , we have the following corollary.

Corollary 6. For $n \in \mathbb{Z}$, we have

(a) $G_{-n}=\frac{1}{t^{n+1}}\left(\left(2 r t-s^{2}\right) G_{n}^{2}+t G_{2 n}+s G_{n+2} G_{n}-(3 t+r s) G_{n+1} G_{n}\right)$.

(b) $H_{-n}=\frac{1}{2 t^{n}}\left(H_{n}^{2}-H_{2 n}\right)$. 
Note that if we take $r=1, s=0, t=1$ and $G_{n}=N_{n}$ in the above Corollary, we obtain Lin's Theorem 1. Using Theorem 2 and Lemma 4 or Remark 5 (or using the last corollary for special cases), we can give some formulas for the special cases of generalized Tribonacci sequence (generalized ( $\mathrm{r}, \mathrm{s}, \mathrm{t})$-sequence) as follows.

We have the following corollary which gives the connection between the special cases of generalized Tribonacci sequence at the positive index and the negative index.

Corollary 7. For $n \in \mathbb{Z}$, we have the following recurrence relations:

(a) Tribonacci sequence:

$$
T_{-n}=T_{n}^{2}+T_{2 n}+T_{n+2} T_{n}-4 T_{n+1} T_{n} .
$$

(b) Tribonacci-Lucas sequence:

$$
K_{-n}=\frac{1}{2}\left(K_{n}^{2}-K_{2 n}\right) .
$$

(c) Tribonacci-Perrin sequence:

$$
\begin{aligned}
& M_{-n}=\frac{1}{3362}\left(243 M_{n+2}^{2}+12 M_{n+1}^{2}+805 M_{n}^{2}-1107 M_{2 n+2}+246 M_{2 n+1}-\right. \\
& \left.943 M_{2 n}-108 M_{n+2} M_{n+1}+1152 M_{n+2} M_{n}-256 M_{n+1} M_{n}\right) .
\end{aligned}
$$

(d) modified Tribonacci sequence:

$$
U_{-n}=\frac{1}{2}\left(U_{n+2}^{2}+4 U_{n+1}^{2}+U_{2 n+2}-2 U_{2 n+1}-4 U_{n+2} U_{n+1}-2 U_{n+2} U_{n}+4 U_{n+1} U_{n}\right) .
$$

(e) modified Tribonacci-Lucas sequence:

$$
\begin{aligned}
& G_{-n}=\frac{1}{2}\left(G_{n+2}^{2}+4 G_{n+1}^{2}-2 G_{2 n+2}+4 G_{2 n+1}-4 G_{n+2} G_{n+1}+G_{n+2} G_{n}-\right. \\
& \left.2 G_{n+1} G_{n}\right) .
\end{aligned}
$$

(f) adjusted Tribonacci-Lucas sequence:

$$
H_{-n}=\frac{1}{2}\left(H_{n+1}^{2}-2 H_{2 n+1}+H_{n+1} H_{n}\right) .
$$

The following corollary illustrates the connection between the special cases of generalized third-order Pell sequence at the positive index and the negative index.

Corollary 8. For $n \in \mathbb{Z}$, we have the following recurrence relations: 
(a) third order Pell sequence:

$$
P_{-n}=3 P_{n}^{2}+P_{2 n}+P_{n+2} P_{n}-5 P_{n+1} P_{n} .
$$

(b) third order Pell-Lucas sequence:

$$
Q_{-n}=\frac{1}{2}\left(Q_{n}^{2}-Q_{2 n}\right) .
$$

(c) third order modified Pell sequence:

$$
E_{-n}=\frac{1}{3}\left(-E_{n}^{2}+3 E_{2 n}-11 E_{n+1} E_{n}+2 E_{n+2} E_{n}\right) .
$$

(d) third order Pell-Perrin sequence:

$$
\begin{aligned}
& R_{-n}=\frac{1}{6962}\left(972 R_{n+2}^{2}+48 R_{n+1}^{2}+1081 R_{n}^{2}-3186 R_{2 n+2}+708 R_{2 n+1}-1357 R_{2 n}-\right. \\
& \left.432 R_{n+2} R_{n+1}+2952 R_{n+2} R_{n}-656 R_{n+1} R_{n}\right) .
\end{aligned}
$$

The following corollary presents the connection between the special cases of generalized Padovan sequence at the positive index and the negative index.

Corollary 9. For $n \in \mathbb{Z}$, we have the following recurrence relations:

(a) Padovan (Cordonnier) sequence:

$$
\begin{aligned}
& P_{-n}=\frac{1}{2}\left(9 P_{n+2}^{2}+4 P_{n+1}^{2}+8 P_{n}^{2}+3 P_{2 n+2}-2 P_{2 n+1}-2 P_{2 n}-12 P_{n+2} P_{n+1}-\right. \\
& \left.18 P_{n+2} P_{n}+12 P_{n+1} P_{n}\right) .
\end{aligned}
$$

(b) Perrin (Padovan-Lucas) sequence:

$$
E_{-n}=\frac{1}{2}\left(E_{n}^{2}-E_{2 n}\right) .
$$

(c) Padovan-Perrin sequence:

$$
S_{-n}=S_{n}^{2}+S_{2 n}-3 S_{n+2} S_{n} .
$$

(d) modified Padovan sequence:

$$
\begin{aligned}
& A_{-n}=\frac{1}{722}\left(3 A_{n+2}^{2}+108 A_{n+1}^{2}+616 A_{n}^{2}+57 A_{2 n+2}+342 A_{2 n+1}-532 A_{2 n}+\right. \\
& \left.36 A_{n+2} A_{n+1}-94 A_{n+2} A_{n}-564 A_{n+1} A_{n}\right) .
\end{aligned}
$$

(e) adjusted Padovan sequence:

$$
U_{-n}=-U_{n}^{2}+U_{2 n}+U_{n+2} U_{n}-3 U_{n+1} U_{n} .
$$


We have the following corollary which gives the connection between the special cases of generalized Pell-Padovan sequence at the positive index and the negative index.

Corollary 10. For $n \in \mathbb{Z}$, we have the following recurrence relations:

(a) Pell-Padovan sequence:

$$
\begin{aligned}
& R_{-n}=\frac{1}{8}\left(16 R_{n+1}^{2}+9 R_{n+2}^{2}+5 R_{n}^{2}+6 R_{2 n+2}-8 R_{2 n+1}-2 R_{2 n}-24 R_{n+2} R_{n+1}-\right. \\
& \left.18 R_{n+2} R_{n}+24 R_{n+1} R_{n}\right) .
\end{aligned}
$$

(b) Pell-Perrin sequence:

$$
\begin{aligned}
& C_{-n}=\frac{1}{242}\left(432 C_{n+2}^{2}+972 C_{n+1}^{2}+665 C_{n}^{2}+396 C_{2 n+2}-594 C_{2 n+1}-385 C_{2 n}-\right. \\
& \left.1296 C_{n+2} C_{n+1}-1104 C_{n+2} C_{n}+1656 C_{n+1} C_{n}\right) .
\end{aligned}
$$

(c) third order Fibonacci-Pell sequence:

$$
\begin{aligned}
& G_{-n}=\frac{1}{2}\left(16 G_{n+2}^{2}+4 G_{n+1}^{2}+35 G_{n}^{2}-4 G_{2 n+2}+2 G_{2 n+1}+7 G_{2 n}-16 G_{n+2} G_{n+1}-\right. \\
& \left.48 G_{n+2} G_{n}+24 G_{n+1} G_{n}\right) .
\end{aligned}
$$

(d) third order Lucas-Pell sequence:

$$
B_{-n}=\frac{1}{2}\left(B_{n}^{2}-B_{2 n}\right) .
$$

(e) adjusted Pell-Padovan sequence:

$$
M_{-n}=-4 M_{n}^{2}+M_{2 n}+2 M_{n+2} M_{n}-3 M_{n+1} M_{n} .
$$

The following corollary illustrates the connection between the special cases of generalized generalized Jacobsthal-Padovan sequence at the positive index and the negative index.

Corollary 11. For $n \in \mathbb{Z}$, we have the following recurrence relations:

(a) Jacobsthal-Padovan sequence:

$$
\begin{aligned}
& Q_{-n}=\frac{1}{2^{n+3}}\left(9 Q_{n+2}^{2}+4 Q_{n+1}^{2}+21 Q_{n}^{2}+6 Q_{2 n+2}-4 Q_{2 n+1}-6 Q_{2 n}-\right. \\
& \left.12 Q_{n+2} Q_{n+1}-30 Q_{n+2} Q_{n}+20 Q_{n+1} Q_{n}\right) .
\end{aligned}
$$


(b) Jacobsthal-Perrin (-Lucas) sequence:

$$
L_{-n}=\frac{1}{2^{n+1}}\left(L_{n}^{2}-L_{2 n}\right) .
$$

(c) adjusted Jacobsthal-Padovan sequence:

$$
K_{-n}=\frac{1}{2^{n+1}}\left(-K_{n}^{2}+2 K_{2 n}+K_{n+2} K_{n}-6 K_{n+1} K_{n}\right) .
$$

(d) modified Jacobsthal-Padovan sequence:

$$
\begin{aligned}
& M_{-n}=\frac{1}{529 \times 2^{n+3}}\left(108 M_{n+1}^{2}+75 M_{n+2}^{2}+3551 M_{n}^{2}+690 M_{2 n+2}+828 M_{2 n+1}-\right. \\
& \left.3082 M_{2 n}+180 M_{n+2} M_{n+1}-1130 M_{n+2} M_{n}-1356 M_{n+1} M_{n}\right) .
\end{aligned}
$$

The following corollary presents the connection between the special cases of generalized Narayana sequence at the positive index and the negative index.

Corollary 12. For $n \in \mathbb{Z}$, we have the following recurrence relations:

(a) Narayana sequence:

$$
N_{-n}=2 N_{n}^{2}+N_{2 n}-3 N_{n+1} N_{n} .
$$

(b) Narayana-Lucas sequence:

$$
U_{-n}=\frac{1}{2}\left(U_{n}^{2}-U_{2 n}\right) .
$$

(c) Narayana-Perrin sequence:

$$
\begin{aligned}
& H_{-n}=\frac{1}{5618}\left(2028 H_{n+1}^{2}+1323 H_{n+2}^{2}+429 H_{n}^{2}-3339 H_{2 n+2}+4134 H_{2 n+1}-\right. \\
& \left.583 H_{2 n}-3276 H_{n+2} H_{n+1}+2688 H_{n+2} H_{n}-3328 H_{n+1} H_{n}\right) .
\end{aligned}
$$

We have the following corollary which gives the connection between the special cases of generalized third-order Jacobsthal sequence at the positive index and the negative index.

Corollary 13. For $n \in \mathbb{Z}$, we have the following recurrence relations:

(a) third order Jacobsthal sequence:

$$
J_{-n}=\frac{1}{2^{n+1}}\left(3 J_{n}^{2}+2 J_{2 n}+J_{n+2} J_{n}-7 J_{n+1} J_{n}\right) .
$$


(b) third order Jacobsthal-Lucas sequence:

$$
\begin{aligned}
& j_{-n}=\frac{1}{9 \times 2^{n+6}}\left(121 j_{n+2}^{2}+441 j_{n+1}^{2}-95 j_{n}^{2}-264 j_{2 n+2}+504 j_{2 n+1}+120 j_{2 n}-\right. \\
& \left.462 j_{n+2} j_{n+1}+154 j_{n+2} j_{n}-294 j_{n+1} j_{n}\right) .
\end{aligned}
$$

(c) modified third order Jacobsthal-Lucas sequence:

$$
K_{-n}=\frac{1}{2^{n+1}}\left(K_{n}^{2}-K_{2 n}\right) .
$$

(d) third order Jacobsthal-Perrin sequence:

$$
\begin{aligned}
& Q_{-n}=\frac{1}{1225 \times 2^{n+3}}\left(243 Q_{n+2}^{2}+3 Q_{n+1}^{2}+3328 Q_{n}^{2}-1890 Q_{2 n+2}+210 Q_{2 n+1}-\right. \\
& \left.3640 Q_{2 n}-54 Q_{n+2} Q_{n+1}+2196 Q_{n+2} Q_{n}-244 Q_{n+1} Q_{n}\right) .
\end{aligned}
$$

The following corollary illustrates the connection between the special cases of generalized generalized 3-primes sequence at the positive index and the negative index.

Corollary 14. For $n \in \mathbb{Z}$, we have the following recurrence relations:

(a) 3-primes sequence:

$$
G_{-n}=\frac{1}{5^{n+1}}\left(11 G_{n}^{2}+5 G_{2 n}+3 G_{n+2} G_{n}-21 G_{n+1} G_{n}\right) .
$$

(b) Lucas 3-primes sequence:

$$
H_{-n}=\frac{1}{2 \times 5^{n}}\left(H_{n}^{2}-H_{2 n}\right) .
$$

(c) modified 3-primes sequence:

$$
E_{-n}=\frac{1}{9 \times 5^{n}}\left(-7 E_{n}^{2}+9 E_{2 n}+4 E_{n+2} E_{n}-31 E_{n+1} E_{n}\right) .
$$

The following corollary presents the connection between the special cases of generalized reverse 3-primes sequence at the positive index and the negative index.

Corollary 15. For $n \in \mathbb{Z}$, we have the following recurrence relations:

(a) reverse 3-primes sequence:

$$
N_{-n}=\frac{1}{2^{n+1}}\left(11 N_{n}^{2}+2 N_{2 n}+3 N_{n+2} N_{n}-21 N_{n+1} N_{n}\right) .
$$


(b) reverse Lucas 3-primes sequence:

$$
S_{-n}=\frac{1}{2^{n+1}}\left(S_{n}^{2}-S_{2 n}\right) .
$$

(c) reverse modified 3-primes sequence:

$$
U_{-n}=\frac{1}{9 \times 2^{n}}\left(-7 U_{n}^{2}+9 U_{2 n}+10 U_{n+2} U_{n}-67 U_{n+1} U_{n}\right) .
$$

\section{References}

[1] I. Bruce, A modified Tribonacci sequence, Fibonacci Quarterly 22(3) (1984), 244-246.

[2] M. Catalani, Identities for Tribonacci-related sequences, 2012. arXiv:math/0209179

[3] E. Choi, Modular Tribonacci numbers by matrix method, Journal of the Korean Society of Mathematical Education Series B: Pure and Applied Mathematics 20(3) (2013), 207-221. https://doi.org/10.7468/jksmeb.2013.20.3.207

[4] M. Elia, Derived sequences, the Tribonacci recurrence and cubic forms, Fibonacci Quarterly 39(2) (2001), 107-115.

[5] M. C. Er, Sums of Fibonacci numbers by matrix methods, Fibonacci Quarterly 22(3) (1984), 204-207.

[6] F. T. Howard, A Tribonacci identity, Fibonacci Quarterly 39(4) (2001), 352-357.

[7] P. Y. Lin, De Moivre-Type identities for the Tribonacci numbers, Fibonacci Quarterly 26 (1988), 131-134.

[8] X. Lin, On the recurrence properties of Narayana's Cows sequence, Symmetry 13 (2021), 149. https://doi.org/10.3390/sym13010149

[9] S. Pethe, Some identities for Tribonacci sequences, Fibonacci Quarterly 26(2) (1988), 144-151.

[10] A. Scott, T. Delaney and V. Hoggatt, Jr., The Tribonacci sequence, Fibonacci Quarterly 15(3) (1977), 193-200.

[11] A. Shannon, Tribonacci numbers and Pascal's pyramid, Fibonacci Quarterly 15(3) (1977), pp. 268 and 275. 
[12] N. J. A. Sloane, The on-line encyclopedia of integer sequences, http://oeis.org/

[13] Y. Soykan, Tribonacci and Tribonacci-Lucas sedenions, Mathematics 7(1) (2019), 74. https://doi.org/10.3390/math7010074

[14] Y. Soykan, On four special cases of generalized Tribonacci sequence: Tribonacci-Perrin, modified Tribonacci, modified Tribonacci-Lucas and adjusted Tribonacci-Lucas sequences, Journal of Progressive Research in Mathematics 16(3) (2020), 3056-3084.

[15] Y. Soykan, On generalized third-order Pell numbers, Asian Journal of Advanced Research and Reports 6(1) (2019), 1-18. https://doi.org/10.9734/ajarr/2019/v6i130144

[16] Y. Soykan, On generalized Padovan numbers, MathLAB Journal, in Print.

[17] Y. Soykan, Generalized Pell-Padovan numbers, Asian Journal of Advanced Research and Reports 11(2) (2020), 8-28.

https://doi.org/10.9734/ajarr/2020/v11i230259

[18] Y. Soykan, A study on generalized Jacobsthal-Padovan numbers, Earthline Journal of Mathematical Sciences 4(2) (2020), 227-251.

https://doi.org/10.34198/ejms.4220.227251

[19] Y. Soykan, On generalized Narayana numbers, Int. J. Adv. Appl. Math. and Mech. $7(3)(2020), 43-56$.

[20] E. E. Polatlı and Y. Soykan, On generalized third-order Jacobsthal numbers, Submitted.

[21] Y. Soykan, On generalized Grahaml numbers, Journal of Advances in Mathematics and Computer Science 35(2) (2020), 42-57.

https://doi.org/10.9734/jamcs/2020/v35i230248

[22] Y. Soykan, A study on generalized $(r, s, t)$-numbers, MathLAB Journal 7 (2020), 101-129.

[23] Y. Soykan, On generalized reverse 3-primes numbers, Journal of Scientific Research and Reports 26(6) (2020), 1-20.

https://doi.org/10.9734/jsrr/2020/v26i630267 
[24] W. Spickerman, Binet's formula for the Tribonacci sequence, Fibonacci Quarterly 20 (1982), 118-120.

[25] C. C. Yalavigi, Properties of Tribonacci numbers, Fibonacci Quarterly 10(3) (1972), 231-246.

This is an open access article distributed under the terms of the Creative Commons Attribution License (http://creativecommons.org/licenses/by/4.0/), which permits unrestricted, use, distribution and reproduction in any medium, or format for any purpose, even commercially provided the work is properly cited. 